\title{
The paediatric story of human papillomavirus (Review)
}

\author{
IOANNIS N. MAMMAS, GEORGE SOURVINOS and DEMETRIOS A. SPANDIDOS \\ Department of Clinical Virology, School of Medicine, University of Crete, Heraklion 71003, Greece
}

Received May 20, 2014; Accepted June 4, 2014

DOI: $10.3892 / \mathrm{ol} .2014 .2226$

\begin{abstract}
Human papillomavirus (HPV) is composed of a particularly heterogeneous family of DNA viruses, which has gained much attention in recent years due to the discoveries of Professor Harald zur Hausen, who first identified a connection between HPV and cervical cancer. Professor Harald zur Hausen, the 'Father of HPV Virology', was the recipient of the 2008 Nobel Prize. HPV can be transmitted through physical contact via autoinoculation or fomites, sexual contact, as well as vertically from the HPV-positive mother to her newborn, causing subclinical or clinical infections. In infancy and childhood, HPV-associated clinical infections include skin warts, genital warts and juvenile recurrent respiratory papillomatosis, while cervical squamous intraepithelial lesions have also been reported among adolescent girls. To date, several research teams, worldwide, have extensively investigated HPV from the paediatric point of view. This primitive effort has been performed before the recent great expansion of paediatric HPV research due to the vaccination programmes against HPV, which were introduced into clinical practice in 2006. In this review article, we present a brief overview of paediatric HPV research after the first report in 1978 involving children in the research of HPV until the time point of this great expansion. In the future, it is expected that further unresolved issues will be addressed and clarified, as the paediatric story of HPV remains a challenging research target.
\end{abstract}

\section{Contents}

1. Introduction

2. Historical background

3. HPV in children: a brief overview

4. Future perspectives

Correspondence to: Professor Demetrios A. Spandidos, Department of Clinical Virology, School of Medicine, University of Crete, Heraklion 71003, Greece

E-mail: spandidos@spandidos.gr

Abbreviations: HPV, human papillomavirus; PCR, polymerase chain reaction; RRP, recurrent respiratory papillomatosis; SILs, squamous intraepithelial lesions; URR, upstream regulatory region

Key words: human papillomavirus, children, lesions, transmission, recurrent respiratory papillomatosis

\section{Introduction}

Human papillomavirus (HPV), the most extensively studied virus of the past decade, is composed of a particularly heterogeneous family of DNA viruses, which has the ability to infect keratinocytes of the human skin and mucosa (1). HPV, which appears to invade the basal layer of epithelial cells, is a common pathogen associated with a wide range of cutaneous and mucosal infections (2). HPV can be transmitted through physical contact via autoinoculation or fomites, sexual contact, as well as vertically from the HPV-positive mother to her newborn and can cause subclinical or clinical infections $(1,2)$. HPV-associated clinical infections include skin warts, genital warts, recurrent respiratory papillomatosis (RRP), low-grade and high-grade squamous intraepithelial lesions (SILs) and cervical cancer, which globally represents the second most frequent cancer in females (3).

In infancy and childhood, HPV infection involving skin warts, genital warts and juvenile RRP among both male and female neonates and children, as well as cervical SILs among adolescent girls (Fig. 1), has been excessively investigated [see reviews by Mammas et al (2) and Syrjänen (4)]. This scientific effort began in 1978, almost 35 years ago, when the first report involving children in HPV research was published by Pfister and zur Hausen (5). To date, several researchers, worldwide, have studied HPV from the paediatric point of view, expanding the usage of molecular techniques, such as the polymerase chain reaction (PCR) in samples obtained from children. During the past years, a great expansion has taken place in the field due to the introduction of the vaccination programmes against HPV into clinical practice. In this review, we briefly summarize some of the historical aspects of peadiatric HPV research until the time point of this great expansion.

\section{Historical background}

HPV-associated lesions, including skin and genital warts, have been reported since ancient times (6). In the 4th century B.C. Hippokrates the Asclepiad, first described skin warts, genital warts and cervical neoplasia (6-8). Although Hippokrates was certainly not the first to discover cervical neoplasia, he referred to a cervical lesion, which in Greek is termed 'ع $\lambda_{\kappa} \kappa_{\varsigma}$ ' (6), meaning 'ulcer' that can potentially progress to cervical cancer, indicating that HPV-associated SILs can progress to invasive cervical cancer. This knowledge referring to the physical history of HPV infection in the cervix is apparent in the impressive description by Hippokrates: ' $\varepsilon$ i 


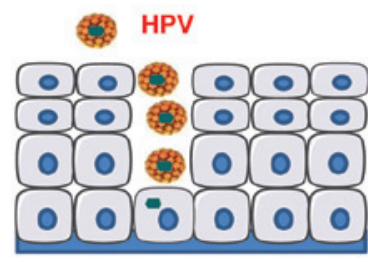

HPV TRANSIENT INFECTION

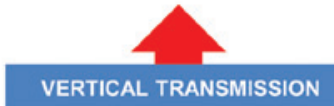

VERTICAL TRANSMISSION

AUTOINOCULATION

SEXUAL TRANSMISSION

TRANSMISSION VIA FOMITES
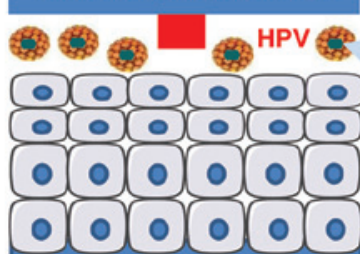

NORMAL EPITHELIUM

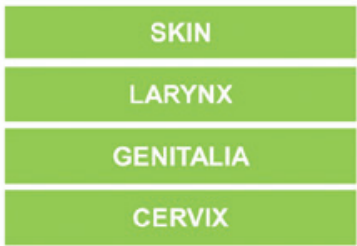

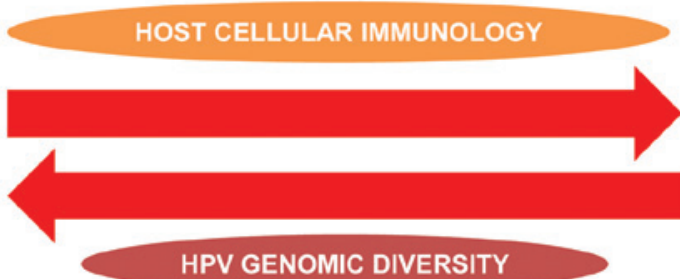

HPV GENOMIC DIVERSITY
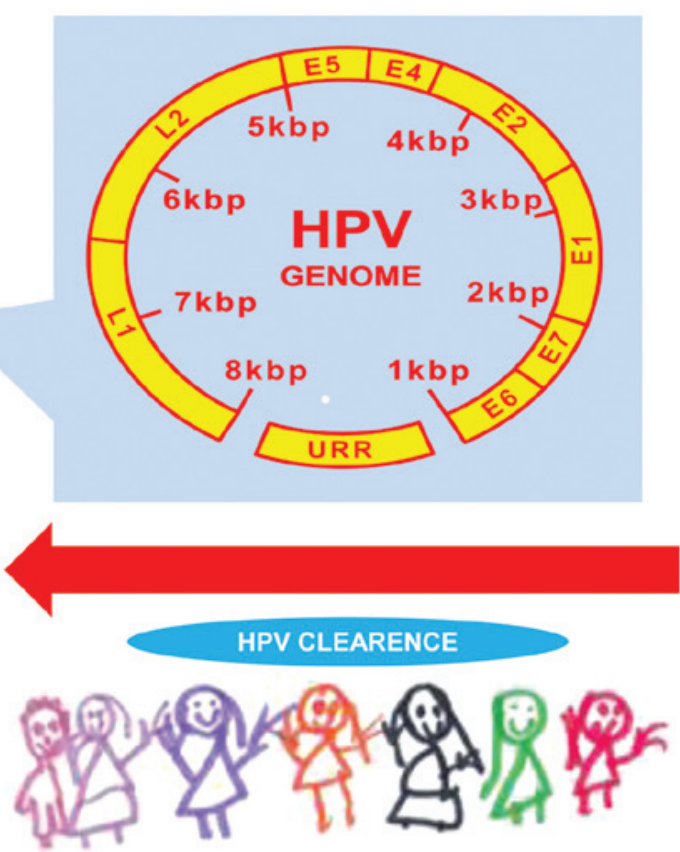

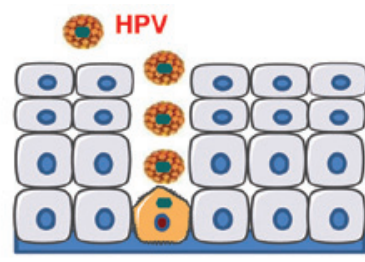

HPV PERSISTENT INFECTION

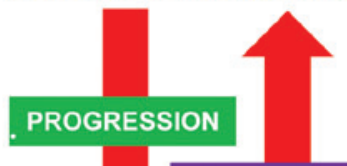

REGRESSION

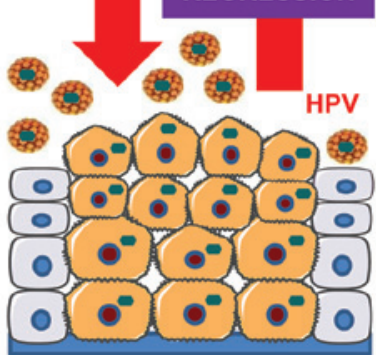

CLINICAL LESIONS

SKIN WARTS

RRP

GENITAL WARTS

CERVICAL SILS

Figure 1. Association between HPV and clinical lesions in neonates and children. HPV can be transmitted via autoinoculation or via fomites, sexual contact or vertically from the HPV-positive mother to her newborn, initially causing HPV transient infection, which can consequently progress to HPV persistent infection. HPV persistence can either regress, or can become symptomatic, establishing clinical lesions in different anatomical sites. In infancy and childhood, HPV clearance can occur automatically. HPV, human papilloma virus; RRV, recurrent respiratory papillomatosis; SILs, squamous intraepithelial lesions; URR, upstream regulatory region.

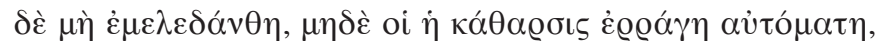

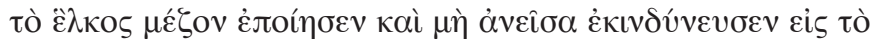
$\kappa \alpha \varrho \varkappa \iota v \omega \theta \hat{\eta} v \alpha \iota ~ \tau \grave{\alpha} \check{\varepsilon} \lambda \kappa \varepsilon \alpha$ ' (7), meaning that 'if it (the infection) is not taken care of, catharsis will not take place automatically, and thus the ulcer will increase in size and if it does not regress, there is a risk of the ulcers becoming cancerous'.

Despite the fact that skin and genital warts have been considered infectious since this early period, the development of cervical cancer due to infection was only suspected in the 19th century A.C. by an Italian scientist from Asiago, Italy, the surgeon Antonio Domenico Rigoni-Stern (9). In 1928, a Greek scientist originating from the island of Euboea, Dr George N. Papanicolaou [a brief referral to his life is presented in the article by Mammas and Spandidos (10)] observed precancerous HPV-associated lesions in vaginal smears collected from females, an observation which led to the development of the Pap smear test $(11,12)$. The first description of HPV was provided in 1949 by Strauss et al (13), who used electron microscopy to examine aqueous extracts of wart tissues, while in 1963 the physical properties of HPV DNA were described in the study by Crawford and Crawford (14). It was not until the 1970s, that a role of HPV in cervical cancer was postulated for the first time by Professor Harald zur Hausen, the 'Father of HPV Virology' (3). It is currently well estab- lished that HPV is involved in human carcinogenesis, causing not only the vast majority of cervical, but also a substantial proportion of other non-genital cancers (15).

\section{HPV in children: a brief overview}

Although the infectious cause of warts in children was known by the end of the 19th century (16), initial studies on children using molecular hybridization techniques were performed in the end of the 1970s. In an early study by Pfister and zur Hausen (5) in 1978, it was well documented that HPV 1, HPV 2 and HPV 3 predominate in skin warts in children between the ages of 5 and 15 years, while HPV 4 is most often isolated in children of older ages. As is presented in Table I, this article was the first in the literature $(5,17-50)$ involving samples obtained from children in HPV research. Evidence of the presence of HPV in juvenile RRP also dates back to the beginning of the 1980s (17-19). These studies have provided strong evidence of the etiology of tumors caused by HPV that was verified by subsequent studies on RRP.

During the second half of the 1980s, a clear picture of the presence of specific types of HPV in genital warts in children was obtained $(21,22,24)$. These initial reports enthusiastically supported HPV typing as an important prognostic tool for 
Table I. HPV pre-vaccination research and children: a brief overview.

\begin{tabular}{|c|c|c|}
\hline Year of publication & Authors/(Refs.) & Contribution \\
\hline 1978 & Pfister and zur Hausen (5) & HPV types in skin warts in children \\
\hline 1981 & Costa et al (17) & HPV types in juvenile RRP \\
\hline 1982 & $\begin{array}{l}\text { Braun et al (18) } \\
\text { Mounts et al (19) }\end{array}$ & $\begin{array}{l}\text { HPV types in juvenile RRP } \\
\text { HPV types in juvenile RRP }\end{array}$ \\
\hline 1986 & $\begin{array}{l}\text { Roman and Fife (20) } \\
\text { Rock et al (21) }\end{array}$ & $\begin{array}{l}\text { HPV types in foreskin in neonates } \\
\text { HPV types in genital warts in children }\end{array}$ \\
\hline 1987 & Vallejos et al (22) & HPV types in genital warts in children \\
\hline 1988 & Steinberg (23) & HPV types in genital warts in children \\
\hline 1989 & $\begin{array}{l}\text { Hanson et al (24) } \\
\text { Sedlacek et al }(25)\end{array}$ & $\begin{array}{l}\text { HPV mother-to-infant transmission } \\
\text { HPV mother-to-infant transmission }\end{array}$ \\
\hline 1990 & $\begin{array}{l}\text { Gibson et al (26) } \\
\text { Padel et al }(27) \\
\text { Jenison } \text { et al }(28)\end{array}$ & $\begin{array}{l}\text { HPV types in genital warts in children } \\
\text { HPV types in genital warts in children } \\
\text { HPV types in oral samples in asymptomatic children }\end{array}$ \\
\hline 1991 & Smith et al (29) & HPV mother-to-infant transmission \\
\hline 1993 & Fredericks et al (30) & HPV mother-to-infant transmission \\
\hline 1994 & $\begin{array}{l}\text { Pakarian et al (31) } \\
\text { Kaye et al (32) }\end{array}$ & $\begin{array}{l}\text { HPV mother-to-infant transmission } \\
\text { HPV viral load as a determinant for mother-to-infant transmission }\end{array}$ \\
\hline 1995 & Cason et al (33) & HPV mother-to-infant transmission \\
\hline 1996 & Alberico et al (34) & HPV mother-to-infant transmission \\
\hline 1998 & Tseng et al (35) & Evaluation of HPV infection and mode of delivery \\
\hline 2000 & Rice et al (36) & HPV types in oral samples in asymptomatic children \\
\hline 2005 & $\begin{array}{l}\text { Rintala et al (37) } \\
\text { Chen et al (38) }\end{array}$ & $\begin{array}{l}\text { HPV mother-to-infant transmission } \\
\text { HPV types in tonsils in asymptomatic children }\end{array}$ \\
\hline 2006 & $\begin{array}{l}\text { Sisk et al (39) } \\
\text { Mammas et al (40) }\end{array}$ & $\begin{array}{l}\text { HPV types in tonsils in asymptomatic children } \\
\text { HPV types in tonsils in asymptomatic children }\end{array}$ \\
\hline 2008 & $\begin{array}{l}\text { Sarkola et al (41) } \\
\text { Mammas et al (42) }\end{array}$ & $\begin{array}{l}\text { Evaluation of HPV types in breast milk } \\
\text { HPV types in skin warts in children }\end{array}$ \\
\hline 2009 & Cazzaniga et al (43) & Evaluation of HPV types in breast milk \\
\hline 2010 & $\begin{array}{l}\text { Mammas et al }(44) \\
\text { Mammas et al }(45) \\
\text { Mammas et al }(46)\end{array}$ & $\begin{array}{l}\text { HPV mother-to-infant transmission } \\
\text { Novel HPV types in juvenile RRP } \\
\text { Evaluation of HPV infection and neonatal prematurity }\end{array}$ \\
\hline 2011 & $\begin{array}{l}\text { Mammas et al }(47) \\
\text { Yoshida et al }(48) \\
\text { Mammas et al }(49)\end{array}$ & $\begin{array}{l}\text { HPV types in lower respiratory tract in children } \\
\text { Evaluation of HPV types in breast milk } \\
\text { Evaluation of HPV types in breast milk }\end{array}$ \\
\hline 2012 & Mammas et al (50) & Evaluation of HPV persistence and mode of delivery \\
\hline
\end{tabular}

HPV, human papillomavirus; RRP, recurrent respiratory papillomatosis.

HPV-infected children, particularly in those infected with HPV 16 and HPV 18, due to the highly oncogenic potential of these two HPV types (24). For this reason, at that time, several paediatric departments were requesting HPV typing in cases with genital warts in order to identify children who were at a risk of developing cancer. At the same time, researchers evaluated the impact of the presence of HPV in the diagnosis of sexual abuse. However, early enough, it was made clear that HPV typing using molecular techniques is not sufficient to determine the source of HPV infection and pursue the possibility of sexual abuse $(24,26,27)$. In the study by Padel et al (27), it was well established that HPV typing does not provide substantial evidence of the presence or absence of sexual transmission.

The presence of HPV DNA in asymptomatic neonates was initially documented in foreskins by Roman and Fife (20). Soon after the report in 1988 by Steinberg (23) addressing the transmission of HPV to the fetus, a number of studies investigated the perinatal modes of HPV transmission in childhood $(25,29)$. These studies supported a vertical transmission mechanism of HPV in children based on the presence of HPV DNA in asymptomatic neonates in oral and genital samples at or shortly after birth (25). The detection of HPV DNA in the amniotic fluid also suggested an in utero mechanism of HPV transmission (25). 
Smith et al provided evidence indicating the prevalence of HPV among pregnant women increases with the gestational age from $8.0 \%$ in the first trimester to $23.1 \%$ in the third trimester (29), while, in 1994, Kaye et al (32) suggested that viral load is a determinant for HPV transmission to the neonate. In the study by Fredericks et al (30) in 1993, it was well established that the contamination of neonates occurs commonly at birth and persists for at least 6 weeks. In a subsequent report in 1995 by Cason et al (33), the authors supported a bimodal distribution of $\operatorname{IgM}$ seropositivity, which peaked between 2 and 5, and 13 and 16 years of age, suggesting that two distinct modes of transmission may occur. Perinatal HPV in infants has also been shown to be related to the mode of delivery and it was suggested that neonates are at a higher risk of exposure to HPV after vaginal delivery than after caesarean delivery (35).

In 1998, a research team from the University of Turku School of Medicine in Finland, initiated the Finnish Family HPV Study, which was the first prospective attempt to assess HPV dynamics at multiple anatomical sites in parents and infants (37). The large number of mother-infant pairs analyzed made it possible to explore the consequences of the presence of HPV in the placenta, umbilical cord blood and breast milk. Studies supporting perinatal HPV transmission have been reviewed by two separate research teams, one at the Department of Virology at Kings College in London, UK (51) and the other at the University of Turku, Finland (52). However, these reports $(51,52)$ have been met with skepticism as regards definitive interpretation. Nevertheless, the potential impact of early acquired HPV neonatal infection on the efficacy of current vaccines for HPV-positive children remains undetermined.

The early findings by Jenison et al (28) in the 1990s that HPV types exist in the oral cavity of asymptomatic children were re-evaluated a decade later. In 2000, Rice et al (36) reported the presence of HPV in oral samples obtained from healthy children, while other researchers documented tonsillar tissue as a reservoir of HPV DNA (38-40). These findings attracted the attention of Reuters Health, raising questions concerning the modes of HPV transmission in childhood (53). Moreover, the presence of HPV in the lower tract in children may be involved in the recent increasing scientific interest of the role of HPV in lung carcinogenesis (47). Despite the detection of HPV DNA in human breast samples (41), it was clarified that this event is rare and there is no contraindication of HPV-positive mothers to breast feed their children $(43,44,48,49)$.

In the following years, our research led out to the detection of novel HPV types, including HPV 13, HPV 39, HPV 40 HPV 56, in juvenile RRP (45). Two more studies (46,50) evaluating HPV infection in relation to neonatal prematurity and the mode of delivery remain unique in the field of pediatric HPV research. The first of these studies (46) did not find any significant evidence that maternal HPV infection is related to neonatal prematurity, while the other study (50) suggested that a caesarean section does not decrease the risk for oral HPV persistence in children. In a recent study, we used for the first time the term 'Trojan horse oncogenic strategy' to describe the physical history of HPV in childhood (54). This hypothesis that children act as a reservoir of silent high risk HPV types, analogous to the Trojan horse in Greek mythology, requires further investigation.

\section{Future perspectives}

Following the approval of the two current vaccines against HPV (55,56), a great expansion of studies involving HPV research and children was observed. These studies aimed to clarify several unresolved issues involving the efficacy and safety of the vaccination programmes against HPV (57-60). Moreover, they attempted to provide evidence to resolve the issues of whether or not the current target ages should be changed, and to determine the necessity of including boys into the vaccination programmes against HPV $(59,60)$. Epidemiological studies aim to determine the factors that influence the participation of adolescents into the vaccination programmes against HPV and propose scheduled strategies to increase this participation. As the vaccination period has already begun, a re-evaluation of the potential modes of HPV transmission in infancy and the physical history of HPV-associated infections in childhood is expected. In the future, further research is required to fully investigate and clarify all these issues, highlighting the fact that the paediatric story of HPV remains a challenging research target for the next generation of researchers. Indeed, the war against HPV continues.

\section{References}

1. zur Hausen H: Papillomaviruses and cancer: from basic studies to clinical application. Nat Rev Cancer 2: 342-350, 2002.

2. Mammas IN, Sourvinos G and Spandidos DA: Human papilloma virus (HPV) infection in children and adolescents. Eur J Pediatr 168: 267-273, 2009.

3. zur Hausen H: Papillomaviruses in the causation of human cancers - a brief historical account. Virology 384: 260-265, 2009.

4. Syrjänen S: Current concepts on human papillomavirus infections in children. APMIS 118: 494-509, 2010.

5. Pfister $\mathrm{H}$ and zur Hausen H: Seroepidemiological studies of human papilloma virus (HPV-1) infections. Int J Cancer 21: 161-165, 1978.

6. Lipourlis D: Hippokrates, Gynaikologia Maieutiki. Zitros Publications, Thessaloniki, 2001

7. Karaberopoulos D: Aristotelis P. Kouzis. O karkinos para tois arxaiois ellisin iatrois. 1902. Stamoulis Publications, Athens, 2004.

8. Gasparini R and Panatto D: Cervical cancer: from Hippocrates through Rigoni-Stern to zur Hausen. Vaccine 27 (Suppl 1): A4-A5, 2009.

9. Rigoni-Stern DA: Fatti statistici relative alle malattie cancerose. Giorn Serv Progr Patol Terap 2: 507-517, 1842.

10. Mammas IN and Spandidos DA: George N. Papanicolaou (1883-1962): Fifty years after the death of a great doctor, scientist and humanitarian. J BUON 17: 180-184, 2012.

11. Papanicolaou GN and Traut HF: The diagnostic value of vaginal smears in carcinoma of the uterus. Am J Obst Gynecol 42: 193-206, 1941.

12. Papanicolaou GN: A new procedure for staining vaginal smears. Science 95: 438-439, 1942.

13. Strauss MJ, Shaw EW, Bunting H and Melnick JL: Crystalline virus-like particles from skin papillomas characterized by intranuclear inclusion bodies. Proc Soc Exp Biol Med 72: 46-50, 1949.

14. Crawford LV and Crawford EM: A comparative study of polyoma and papilloma viruses. Virology 21: 258-263, 1963.

15. Mammas IN, Sourvinos G, Zaravinos A and Spandidos DA: Vaccination against human papilloma virus (HPV): epidemiological evidence of HPV in non-genital cancers. Pathol Oncol Res 17: 103-119, 2011.

16. Payne J: On the contagious rise of common warts. Br J Dermatol 3: 185, 1891

17. Costa J, Howley PM, Bowling MC, Howard R and Bauer WC: Presence of human papilloma viral antigens in juvenile multiple laryngeal papilloma. Am J Clin Pathol 75: 194-197, 1981. 
18. Braun L, Kashima H, Eggleston J and Shah K: Demonstration of papillomavirus antigen in paraffin sections of laryngeal papillomas. Laryngoscope 92: 640-643, 1982.

19. Mounts P, Shah KV and Kashima H: Viral etiology of juvenileand adult-onset squamous papilloma of the larynx. Proc Nat Acad Sci USA 79: 5425-5429, 1982.

20. Roman A and Fife K: Human papillomavirus DNA associated with foreskins of normal newborns. J Infect Dis 153: 855-861, 1986.

21. Rock B, Naghashfar Z, Barnett N, Buscema J, Woodruff JD and Shah K: Genital tract papillomavirus infection in children. Arch Dermatol 122: 1129-1132, 1986.

22. Vallejos H, Del Mistro A, Kleinhaus S, Braunstein JD, Halwer M and Koss LG: Characterization of human papilloma virus types in condylomata acuminata in children by in situ hybridization. Lab Invest 56: 611-615, 1987.

23. Steinberg BM: Papillomavirus. Effects upon mother and child Ann N Y Acad Sci 549: 118-128, 1988.

24. Hanson RM, Glasson M, McCrossin I, Rogers M, Rose B and Thompson C: Anogenital warts in childhood. Child Abuse Negl 13: 225-233, 1989.

25. Sedlacek TV, Lindheim S, Eder C, Hasty L, Woodland M, Ludomirsky A and Rando RF: Mechanism for human papillomavirus transmission at birth. Am J Obstet Gynecol 161: 55-59, 1989.

26. Gibson PE, Gardner SD and Best SJ: Human papillomavirus types in anogenital warts of children. J Med Virol 30: 142-145, 1990.

27. Padel AF, Venning VA, Evans MF, Quantrill AM and Fleming KA Human papillomaviruses in anogenital warts in children: typing by in situ hybridisation. BMJ 300: 1491-1494, 1990.

28. Jenison SA, Yu XP, Valentine JM, Koutsky LA, Christiansen AE, Beckmann AM and Galloway DA: Evidence of prevalent genitaltype human papillomavirus infections in adults and children. J Infect Dis 162: 60-69, 1990.

29. Smith EM, Johnson SR, Jiang D, et al: The association between pregnancy and human papilloma virus prevalence. Cancer Detect Prev 15: 397-402, 1991.

30. Fredericks BD, Balkin A, Daniel HW, Schonrock J, Ward B and Frazer IH: Transmission of human papillomaviruses from mother to child. Aust N Z J Obstet Gynaecol 33: 30-32, 1993.

31. Pakarian F, Kaye JN, Cason J, et al: Cancer associated human papillomaviruses: perinatal transmission and persistence. Br J Obstet Gynaecol 101: 514-517, 1994.

32. Kaye JN, Cason J, Pakarian FB, et al: Viral load as a determinant for transmission of human papillomavirus type 16 from mother to child. J Med Virol 44: 415-421, 1994.

33. Cason J, Kaye JN, Jewers RJ, et al: Perinatal infection and persistence of human papillomavirus types 16 and 18 in infants. J Med Virol 47: 209-218, 1995.

34. Alberico S, Pinzano R, Comar M, Toffoletti F, Maso G, Ricci G and Guaschino S: Maternal-fetal transmission of human papillomavirus. Minerva Ginecol 48: 199-204, 1996.

35. Tseng CJ, Liang CC, Soong YK and Pao CC: Perinatal transmission of human papillomavirus in infants: relationship between infection rate and mode of delivery. Obstet Gynecol 91: 92-96, 1998.

36. Rice PS, Mant C, Cason J, Bible JM, Muir P, Kell B and Best JM: High prevalence of human papillomavirus type 16 infection among children. J Med Virol 61: 70-75, 2000.

37. Rintala MA, Grénman SE, Puranen MH, Isolauri E, Ekblad U, Kero PO and Syrjänen SM: Transmission of high-risk human papillomavirus (HPV) between parents and infant: a prospective study of HPV in families in Finland. J Clin Microbiol 43: 376-381, 2005.

38. Chen R, Sehr P, Waterboer T, Leivo I, Pawlita M, Vaheri A and Aaltonen LM: Presence of DNA of human papillomavirus 16 but no other types in tumor-free tonsillar tissue. J Clin Microbiol 43 : $1408-1410,2005$

39. Sisk J, Schweinfurth JM, Wang XT and Chong K: Presence of human papillomavirus DNA in tonsillectomy specimens. Laryngoscope 116: 1372-1374, 2006.
40. Mammas IN, Sourvinos G, Michael C and Spandidos DA: Human papilloma virus in hyperplastic tonsillar and adenoid tissues in children. Pediatr Infect Dis J 25: 1158-1162, 2006.

41. Sarkola M, Rintala M, Grénman S and Syrjänen S: Human papillomavirus DNA detected in breast milk. Pediatr Infect Dis J 27: $557-558,2008$

42. Mammas I, Sourvinos G, Michael C and Spandidos DA High-risk human papilloma viruses (HPVs) were not detected in the benign skin lesions of a small number of children. Acta Paediatr 97: 1669-1671, 2008.

43. Cazzaniga M, Gheit T, Casadio C, et al: Analysis of the presence of cutaneous and mucosal papillomavirus types in ductal lavage fluid, milk and colostrum to evaluate its role in breast carcinogenesis. Breast Cancer Res Treat 114: 599-605, 2009.

44. Mammas IN and Spandidos DA: No evidence of mother-to-infant transmission of human papilloma virus via human breast milk. Pediatr Infect Dis J 29: 93, 2010.

45. Mammas IN, Sourvinos G, Vakonaki E, Giamarelou P, Michael C and Spandidos DA: Novel human papilloma virus (HPV) genotypes in children with recurrent respiratory papillomatosis. Eur J Pediatr 169: 1017-1021, 2010.

46. Mammas IN, Sourvinos G and Spandidos DA: Maternal human papillomavirus (HPV) infection and its possible relationship with neonatal prematurity. Br J Biomed Sci 67: 222-224, 2010.

47. Mammas IN, Zaravinos A, Sourvinos G and Spandidos DA Detection of human papillomavirus in bronchoalveolar lavage samples in immunocompetent children. Pediatr Infect Dis J 30: 384-386, 2011.

48. Yoshida K, Furumoto H, Abe A, et al: The possibility of vertical transmission of human papillomavirus through maternal milk. J Obstet Gynaecol 31: 503-506, 2011.

49. Mammas IN, Zaravinos A, Sourvinos G, Myriokefalitakis N, Theodoridou M and Spandidos DA: Can 'high-risk' human papillomaviruses (HPVs) be detected in human breast milk? Acta Paediatr 100: 705-707, 2011

50. Mammas IN, Sourvinos G, Giamarelou P, Michael C and Spandidos DA: Human papillomavirus in the oral cavity of children and mode of delivery: a retrospective study. Int J STD AIDS 23: 185-188, 2012.

51. Cason J, Rice P and Best JM: Transmission of cervical cancerassociated human papilloma viruses from mother to child. Intervirology 41: 213-218, 1998.

52. Syrjänen S and Puranen M: Human papillomavirus infections in children: the potential role of maternal transmission. Crit Rev Oral Biol Med 11: 259-274, 2000.

53. Boggs W: Human papilloma virus present in hyperplastic tonsillar tissue in children. Reuters Health: December 28, 2006 at www.reuters.com.

54. Mammas IN, Sourvinos G and Spandidos DA: The 'Trojan horse' oncogenic strategy of HPVs in childhood. Fut Virology 8: 801-808, 2013

55. FUTURE II Study Group: Quadrivalent vaccine against human papillomavirus to prevent high-grade cervical lesions. N Engl J Med 356: 1915-1927, 2007.

56. Paavonen J, Naud P, Salmerón J, et al: Efficacy of human papillomavirus (HPV)-16/18 AS04-adjuvanted vaccine against cervical infection and precancer caused by oncogenic HPV types (PATRICIA): final analysis of a double-blind, randomised study in young women. Lancet 374: 301-314, 2009.

57. Rafle AE: Challenges of implementing human papillomavirus (HPV) vaccination policy. BMJ 335: 375-377, 2007.

58. Maher F and Mammas I: HPV vaccination in younger preadolescents? 13 September 2007 at www.bmj.com/content/335/ $7616 / 375 ? \mathrm{tab}=$ responses

59. Mammas I, Maher F, Theodoridou M and Spandidos DA: Human papilloma virus (HPV) vaccination in childhood: challenges and perspectives. Hippokratia 15: 299-303, 2011.

60. Mammas IN and Spandidos DA: Vaccination against human papillomavirus in childhood: the next rubella analogue? J BUON 17: 389-390, 2012. 